
allemande

50-2 | 2018

Humanités environnementales - Quoi de neuf du côté des méthodes?

\title{
Le croquis ethnographique, du regard au trait sur le papier
}

Retour d'expérience d'un atelier étudiant

Chloé Le Mouël et Lucille Maugez

\section{(2) OpenEdition \\ Journals}

\section{Édition électronique}

URL : https://journals.openedition.org/allemagne/856

DOI : 10.4000/allemagne.856

ISSN : 2605-7913

\section{Éditeur}

Société d'études allemandes

\section{Édition imprimée}

Date de publication : 30 décembre 2018

Pagination : 255-266

ISSN : 0035-0974

Référence électronique

Chloé Le Mouël et Lucille Maugez, "Le croquis ethnographique, du regard au trait sur le papier », Revue d'Allemagne et des pays de langue allemande [En ligne], 50-2 | 2018, mis en ligne le 30 décembre 2019, consulté le 19 mai 2021. URL : http://journals.openedition.org/allemagne/856 ; DOI : https:// doi.org/10.4000/allemagne.856 


\title{
Le croquis ethnographique, du regard au trait sur le papier Retour d'expérience d'un atelier étudiant
}

\author{
- Chloé Le Mouël*, Lucille Maugez**
}

Un atelier de croquis ethnographique a été mis en place, sur une initiative étudiante, à l'Institut d'ethnologie de l'université de Strasbourg au courant de l'année scolaire 2016/2017. Nous proposons ici de retracer un retour d'expérience de cette activité. Le projet a été créé à l'initiative de Lucille Maugez, étudiante en master anthropologie sociale et culturelle et présidente de l'Association d'ethnologie de Strasbourg, structure ayant encadré la mise en place de ce projet. Il est né de la rencontre de son désir de trouver une alternative à la photographie, méthode peu adaptée à son terrain, et de l'envie de Laurane Delavier et Chloé Le Mouël de créer un lieu de réflexion et de transmission de connaissance autour d'une méthode qu'elles utilisaient intuitivement. Conjointement, nous avons décidé de mener à bien ce projet afin d'enrichir notre formation et de transmettre les connaissances que nous avions en relation à la pratique du dessin.

Cet atelier a été construit au cours de dix séances de deux heures pensées comme le lieu d'une expérimentation collective. Nous avons suivi comme fil rouge le «corps dans l'espace ", thème volontairement ample rendant possible l'intégration de concepts empruntés à d'autres disciplines. Cette multidisciplinarité a été permise par la pluralité des formations des intervenantes, en anthropologie sociale et culturelle, illustration, architecture et archéologie. Ainsi, nous avions des connaissances complémentaires quant au dessin, à l'anthropologie et à nos domaines de spécialité. En conséquence, nous nous sommes placées à la fois comme animatrices et comme participantes de l'atelier; ce retour d'expérience reflète cette double position. L'atelier était destiné aux

* Doctorante en architecture et anthropologie, Laboratoire Amup, Université de Strasbourg; Laboratoire Ciéra, Université Laval.

** Étudiante en master anthropologie sociale et culturelle, Institut d'ethnologie, Université de Strasbourg, présidente de l'Association d'ethnologie de Strasbourg 2016-2017. 
élèves d'anthropologie sociale et culturelle, mais il a également été ouvert à toute personne intéressée. Cela a permis d'accueillir des élèves en sociologie, en géographie, en graphisme ou encore en archéologie. Surtout, il était ouvert aux débutants comme aux personnes ayant déjà une grande maîtrise du dessin. Cette diversité de regards et de pratiques de la part de tous les acteurs a été une vraie richesse pour l'atelier de croquis. Elle nous a notamment amenés à réfléchir sur les qualités prêtées au dessin ethnographique et à son utilisation comme outil d'enquête. Nous avons par conséquent discuté autour de la question esthétique du dessin, nous demandant si ce dernier pouvait être utilisé comme outil d'enquête lorsque nous ne savions pas dessiner. Ce débat venait du fait que la première réaction de nombreux participants était: «Ce n'est pas pour moi, je ne sais pas dessiner. » Nous avons par la suite élargi notre problématique à l'utilisation du dessin comme mode de production valide du savoir. Nous avons exploré une utilisation du croquis comme prise de notes sur le terrain dans le carnet de recherche, comme alternative à la prise de note écrite, visant à produire du matériel utilisable pour l'analyse.

Les sujets développés au cours des séances n’ont pas été planifiés en amont mais ont répondu aux besoins et aux envies de chacun, et se sont structurés au gré de nos lectures et des questions soulevées lors de nos rencontres. Ces séances se sont tenues dans différents lieux afin de varier la dynamique et le positionnement observateurobservé: salles de cours de l'université, musée, campus, brasserie, places publiques, etc. Cette expérience a été clôturée par la mise en place d'une exposition au sein de la Maison universitaire internationale en date du 2 juin 2017. Celle-ci avait pour objectif de valoriser le travail des participants et de porter la visibilité de l'usage du dessin en anthropologie en dehors de la scène universitaire. Les croquis exposés à cette occasion ont été choisis pour leur qualité didactique. Ils ont été accompagnés de planches de bandes dessinées réalisées par Laurane Delavier, reprenant les divers thèmes abordés au cours de l'année, ainsi que de photographies prises au cours des séances permettant d'illustrer l'expérience vécue par les participants.

Avant d'entrer dans le vif du sujet, nous proposons au lecteur d'interrompre sa lecture un instant afin d'effectuer un rapide exercice qui lui permettra de s'approprier davantage les réflexions que nous allons développer. Nous vous proposons de vous installer confortablement et de prendre un crayon. Choisissez autour de vous une personne, si celle-ci n'est pas en mouvement l'exercice n'en sera que plus simple. La consigne est la suivante: dessinez cette personne en 1 minute, puis en 30 secondes, puis en 10 secondes. Vous avez ainsi pu vous rendre compte que le regard que vous avez porté sur la scène n'était plus le même. Vous avez également dû faire des choix sur le contenu représenté : la posture, les vêtements, la relation au contexte, à la lumière? Quel(s) outil(s), quel support avez-vous choisi, et comment avez-vous placé le dessin sur le support? Que faire quand le modèle bouge? Ce sont ces types de questions que l'atelier de croquis a permis d'explorer collectivement. Cet article reprend et prolonge certaines réflexions menées par le groupe, notamment sous l'angle du croquis comme regard spécifique sur le monde et comme outil de communication. 


\section{Le dessin comme méthode anthropologique}

La relation entre le dessin et l'anthropologie est visible depuis le XIX ${ }^{\mathrm{e}}$ siècle, au moment où celle-ci s'est constituée comme discipline indépendante. L'utilisation du graphisme avait alors pour fonctions d'illustrer et de créer des registres des éléments de la culture matérielle et des observations anatomiques faites des populations par les ethnographes. Au cours du siècle suivant, les anthropologues ont cherché à se détacher de ces objets de recherche, ceux-ci étant associés à la pratique du collectionnisme et au folklorisme, conduisant dans le même temps à délaisser le dessin comme forme complémentaire de l'écrit ${ }^{(1)}$. Pour Aina Azevedo ${ }^{(2)}$ ce phénomène a été accentué par l'avènement de la photographie et du cinéma, supports qui ont été privilégiés par la discipline. Cela s'est traduit par leur institutionnalisation comme outils d'enquêtes et par la constitution de l'anthropologie visuelle dont le dessin fut écarté. À l'exception de la Russie, unique exemple qui nous est parvenu ${ }^{(3)}$, le support graphique n'a pas été développé comme méthode spécifique. Il demeure par conséquent un concept vague dont la réalisation dépend des conventions qu'il emprunte à d'autres domaines d'étude. Le dessin n'est donc pas par définition anthropologique, il le «devient au travers des modes de pensée, de voir et d'interpréter du dessinateur ainsi que de comprendre le monde par le biais de l'anthropologie» ${ }^{(4)}$. Nous pouvons cependant noter une inversion de ce processus au cours des dernières années, moment qualifié de "virée graphique» par Chris Ballard ${ }^{(5)}$. Cela se traduit par la multiplication de publications intégrant le dessin et appréhendant son utilisation. Cet événement peut être pensé dans un contexte de réinvention de la production anthropologique passant notamment par la recherche de nouvelles valeurs telles que la qualité "sensible» ${ }^{(6)}$. Nombre de ces travaux ont été référencés et étudiés par Aina Azevedo ${ }^{(7)}$, exposant les possibilités qu'offre la pratique du dessin. Celui-ci apparaît tantôt comme méthodologie, comme un substitut du discours narré, comme résultat d'étude et une forme de les présenter, comme un apport stylistique. En somme, ces travaux mettent en lumière une appropriation du dessin comme support analytique, rôle allant au-delà de ceux de témoins, de registres et d'observations que leur attribua Marcel Mauss ${ }^{(8)}$.

1 João LEAL, «Retratos do povo etnografia portuguesa e imagem», in: J. MACHAdo PAIS, C. CARVAlho et N.M. Gusmão (dir.), O visual e o quotidiano, Lisbonne, Imprensa de Ciências Sociais, 2008, p. 117-145.

2 Aina Azevedo, «Diário de campo e diário gráfico: contribuições do desenho à antropologia», Revista de Antropologia, 2/2 (2016), p. 100-119, ici p. 103.

3 Haidy Geismar, «Drawing it Out», Visual Anthropological Review, nº 30 (2014), p. 96-113.

4 Gomes, cité par Karina Kuschnir, «A antropologia pelo desenho: experiências visuais e etnográficas», Cadernos de Arte e Antropologia, 5/2 (2016), p. 5-13, ici p. 11.

5 Chris BAllard, «The Return of the Past: On Drawing and Dialogic History ", The Asia Pacific Journal of Anthropology, n 14/2 (2013), p. 136-148, ici p. 140.

6 K. Kuschnir, "A antropologia pelo desenho» (note 4).

7 Aina Azevedo, «Desenho e antropologia : recuperação histórica e momento atual », Cadernos de Arte e Antropologia, 5/2 (2016), p. 15-32, ici p. 22.

8 Marcel Mauss, Manuel d'ethnographie, Paris, Payot, 1967. 


\section{Apprendre à voir et à penser le monde}

C'est cette dimension analytique que nous avons cherché à explorer au cours de nos séances. Afin de l'aborder, nous avons dû affronter les appréhensions des participants, essentiellement construites sur l'idée que le dessin doit être beau pour être exploitable. Pour une partie d'entre eux, l'atelier représentait une première expérience graphique, créant un certain complexe et une réticence à montrer leurs productions. Nous avons par conséquent construit notre discours en valorisant le processus d'élaboration en lui-même et en écartant la dimension esthétique qui nous est apparue secondaire. Comme l'évoque John Berger ${ }^{(9)}$, «ce qui est en jeu ce n'est pas le résultat, à savoir la ligne dessinée, mais le processus par lequel le dessinateur passe pour voir et connaître à partir du tracé sur le papier ».

Cet atelier nous a ainsi permis de vivre «l'expérience du dessin ${ }^{(10)}$, traduite par une prise de conscience qu'il existe diverses manières d'observer le monde. Nous pouvons le voir, à savoir être témoin d'une scène perçue, ou le regarder, c'est-à-dire l'aborder au travers d'une observation et d'une réflexion, afin de devenir acteur de ce monde. Le dessin s'inscrit au sein de ce second mode. Le dessinateur est en effet intégré à la scène qu'il observe en la transcrivant sur un support, vu comme un pont entre deux mondes, celui du dessin et celui de l'ethnographe, "tous deux immergés dans un voyage recherchant l'observation et l'expérience dans un quotidien étranger ${ }^{(11)}$. Cette retranscription évoque donc une nouvelle appréhension du monde, mettant en relation un regard et un mode de pensée particuliers où l'acte de penser est associé à celui de faire, construisant une «connaissance au travers du faire» et du «connaître depuis le dedans ${ }^{(12)}$. Le dessin n'évoque pas une simple projection d'une idée sur le papier ni la narration faite a posteriori mais surgit conjointement à ce qui est observé(13), établissant une connexion entre cet élément et sa description ${ }^{(14)}$. Cela fait écho à la méthode de l'observation participante durant laquelle le chercheur est amené à reproduire les pratiques de ses interlocuteurs, lui permettant d'étendre la réflexion et la compréhension qu'il porte sur celles-ci. Ce support retrace ainsi l'expérience vécue par l'observateur, sortant du cadre du visible pour intégrer des éléments de l'ordre du sensible.

Cette transcription présuppose ainsi une transformation de l'information par le biais des choix opérés par le dessinateur. Celui-ci va ainsi s’approprier le tracé selon les codes et les techniques qui font sens pour lui, fait que nous détaillerons dans la suite de l'article. Par conséquent, le dessin peut être pensé comme un support illustrant la subjectivité de celui qui lui donne forme. Il n'est donc pas neutre car le regard du dessinateur ne l'est pas. Regarder dans le contexte ethnographique est compris comme une capacité et conduit à un apprentissage. C’est en cela que réside la difficulté du dessin,

9 John Berger, Berger on Drawing, Aghabullogue, Occasional Press, 2005, ici p. 70.

10 K. Kuschnir, «A antropologia pelo desenho» (note 4).

11 Ibid., p. 8.

12 Tim Ingold, Being Alive. Essays on Movement, Knowledge and Description, Londres/New-York, Routledge, 2011; ID., Redrawing Anthropology. Materials, Movements, Lines, Ashgate (Angleterre), 2011.

13 Tim Ingold, Making, Anthropology, Archeology, Art and Architecture, Londres/New-York, Routledge, 2013, ici p. 126-129.

14 T. Ingold, Redrawing Anthropology (note 12), p. 9. 
exercice basé sur une habileté visuelle plus que manuelle ${ }^{(15)}$. Cet apprentissage pour l'auteur amène à se détacher de nos perceptions liées à la modalité associant la raison à la capacité verbale afin de les traduire de façon non-verbale et à l'élaboration de ce que Roberto Cardoso de Oliveira ${ }^{(16)}$ nomme le «regard ethnographique». Il est le fruit d'observations minutieuses et prolongées qui permettent de mettre en évidence les détails de la scène. Ce fait est particulièrement perceptible en contexte familier au sein duquel la cohabitation prolongée conduit à l'effacement du détail. Le dessin ici permet de rendre visible ce qui était demeuré invisible ou ce qui l'était devenu.

Ce point a été soulevé par nos participants lors de notre atelier au Musée zoologique de Strasbourg au sein duquel nous souhaitions observer les modalités de déplacement du public et ses positionnements face aux vitrines. La faible affluence ce jour-là nous a poussé à «croquer» les animaux exposés. Du fait de sa proximité avec l'université de Strasbourg, le Musée zoologique est un espace privilégié par les étudiants lors de sorties culturelles. Nombre des participants présents lors de cette séance avaient ainsi eu l'occasion de fréquenter cet espace de manière régulière, nous y compris. Nous pensions de ce fait avoir une bonne connaissance de celui-ci et des pièces de sa collection. Cependant, nous nous sommes rendus compte que l'observation que nous avons portée sur les spécimens exposés allait au-delà de celle s'inscrivant dans un contexte de visite classique. En s'attardant sur un sujet et en tentant de le reproduire, nous avons mis en lumière des détails qui étaient jusque-là passés inaperçus. Plusieurs participants ont ainsi déclaré que cette séance leur avait permis de redécouvrir le musée au travers d'un regard nouveau.

\section{Apprendre à signifier}

Le croquis ethnographique est donc une trace laissée sur le papier que le chercheur s'adresse à lui-même pour retranscrire une expérience vécue. Ainsi, le croquis réalisé est un signe, et possède une signification. En sémiotique, selon Charles Peirce ${ }^{(17)}$, un signe se compose d'un representamen, c'est-à-dire un signe matériel, dans notre cas un croquis; d'un objet, la situation ou l'élément observé par le chercheur; d'un processus appelé interprétant reliant les deux premiers éléments, qui est la représentation mentale de la relation entre les deux. Peirce distingue trois types de signes : l'icône, l'indice et le symbole ${ }^{(18)}$. L'icône est un representamen associé à son objet par ressemblance, l'indice est une trace directe laissée par l'objet, telle une trace de pas dans la neige, enfin pour le symbole l'association se fait par convention. Les croquis réalisés lors des ateliers se situent dans un continuum entre icône et symbole. Certaines images sont construites en hybridant icônes et symboles, permettant d'ajouter de la précision. Ainsi, sur les croquis de la chaîne opératoire de la fabrication d'un chapeau en palme, le couteau et la palme sont des icônes, les flèches indiquant les mouvements sont des symboles. Le bonhomme-bâton, quant à lui, se situe entre ces deux registres: le trait cherchant

15 Betty Edwards, Desenhando com o lado direito do cérebro (1979), Rio de Janeiro, Ediouro, 2001.

16 Roberto Cardoso de Oliveira, «O trabalho do antropólogo: olhar, ouvir, escrever», O trabalho do antropólogo, São Paulo, Unesp, 2000 (2 éd.), p. 17-35.

17 Charles Sanders Peirce, Collected Papers, Volumes I and II, Harvard University Press, 1960, p. 141.

18 Ibid., p. 143. 
l'efficacité tend à devenir abstrait. Ces croquis forment un système de signes lisibles, dont l'organisation dans la feuille organise la lecture et informe sur la chronologie.

1. Chaîne opératoire de la confection d'un chapeau en palme
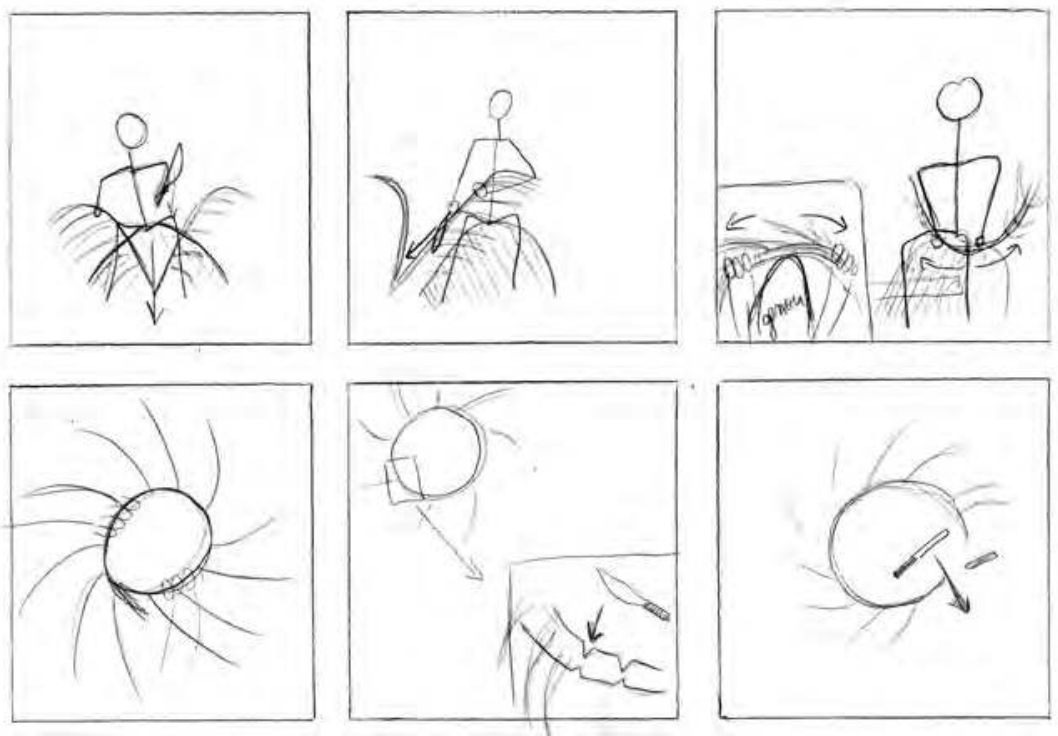

Néanmoins, aucun croquis ne peut se revendiquer d'être un indice, contrairement à la photographie, produite automatiquement et directement déterminée par son référent, qui est une trace directe du réel selon Albert Piette. Or, pour lui, c'est cette qualité indiciaire qui donne à la photographie une valeur documentaire ${ }^{(19)}$. Il oppose en cela la photographie au dessin, refusant par la même occasion à ce dernier toute prétention à un usage scientifique. Or, nous soutenons l'hypothèse contraire: que le croquis ethnographique est un mode de production du savoir valide. Une icône est similaire à son objet, mais n'en est pas une copie conforme. Elle a la capacité à ressembler à ce qu'elle désigne, mais diffère profondément de son objet. Les croquis ne cherchent pas à imiter le réel: ils cherchent à en extraire un ou plusieurs éléments, et à les hiérarchiser. Le croquis résulte d'une suite de choix dans ses trois temps: observer, sélectionner une information, la retranscrire. Il dépouille la scène du superflu et informe du regard qu'a porté le dessinateur sur la scène observée. Le regard du chercheur est guidé par la visée de connaissance, comme nous l'avons vu précédemment. La force du croquis réside dans le fait qu'il contient la scène et la lecture de la scène. En cela, il n'est pas une reproduction de la réalité mais une hypothèse portée par le chercheur ${ }^{(20)}$.

19 Albert Piette, «Fondements épistémologiques de la photographie», Ethnologie française, vol. 37 (2007), p. 23-28, ici p. 25.

20 Christine EsCALlier, «De l'objet intrinsèque à la pensée technique: le rôle médiateur du dessin en ethnographie maritime», Cadernos de Arte e Antropologia, 5/2 (2016), p. 49-73, ici p. 71. 


\section{Apprendre à jouer avec les conventions}

Ainsi, la relation entre le croquis et sa signification prime sur la recherche de l'esthétique. Le dessinateur s'adresse avant tout à lui-même et utilise le croquis comme activateur de mémoire. Pour cela, il doit se souvenir des conventions choisies pour «décoder» le message, avoir accès à l'objet. Il s'agit de codes entrant dans le panel de références du dessinateur. Connaître différentes conventions de représentation permet de jouer avec elles, et de passer de l'une à l'autre selon leurs pertinences. Cela permet de prendre du recul sur l'évidence de l'utilisation de la perspective. Comme l'a montré Erwin Panofsky ${ }^{(21)}$, la perspective n'est pas une loi universelle de la représentation de l'espace, elle n'est pas non plus une restitution fidèle de la vision humaine, mais un code culturel, une forme symbolique employée pour sa valeur signifiante. Ainsi au cours des séances nous avons porté une grande attention au fait d'explorer et tester différentes conventions de représentation. Par exemple, nous avons appris à réaliser des relevés ethno-architecturaux, méthode utilisée par Daniel Pinson ${ }^{(22)}$, architecte et sociologue, qui croise des apports de ces deux disciplines. Ces relevés s'appuient sur le plan, la coupe et l'élévation, qui sont des outils communs pour les architectes. Le plan ne correspond pas à une réalité telle qu'elle est perçue par l'œil humain, mais une représentation de la réalité selon une convention qui veut que l'on coupe à un mètre du sol et que soit représenté, à une échelle donnée, dans le plan du papier, tout ce qui est visible en dessous de cette ligne de coupe fictive. Une légende permet de comprendre les symboles : différentes hachures selon les matériaux, symboles géométriques pour les éléments techniques, etc. Mais les relevés ethno-architecturaux se distinguent des relevés architecturaux par leur but: ces derniers doivent faire l'état des lieux d'une situation pour en projeter le futur, les relevés ethno-architecturaux ont pour but de mettre en évidence la relation entre l'espace et les pratiques sociales ${ }^{(23)}$. Pour cela, ils portent en plus les indications des objets et leur emplacement qui informent sur le mode de vie des occupants. Ainsi, cette méthode de croquis reprend les conventions de retranscription issues de l'architecture et permet d'analyser des espaces construits et de leurs usages. Elle permet d'observer l'habiter comme prise de possession et marquage des lieux ${ }^{(24)}$. Mais elle ne rend pas compte des représentations subjectives liées à un espace, comme les cartes mentales peuvent le faire ${ }^{(25)}$. Ces dernières sont composées de points de repère, de réseaux, de zones, de frontières, de nœuds. Elles ne font pas appel à une bibliothèque pré-établie et partagée par toute une profession : chacun invente ses propres codes au fur et à mesure de la construction de sa carte. Lors de l'atelier dédié aux cartes mentales, nous avons constaté l'invocation de codes culturels: un symbole de petite maison pour indiquer l'habitation même quand celle-ci est un appartement, ou encore des vaguelettes pour symboliser la rivière.

21 Erwin PANOfSky, La perspective comme forme symbolique et autres essais, Paris, Minuit, 1975.

22 Daniel Pinson, «L'habitat, relevé et révélé par le dessin: observer l'espace construit et son appropriation ", Espaces et sociétés, $\mathrm{n}^{\circ}$ 164-165 (2016), p. 49-66, ici p. 55.

23 Ibid., p. 57.

24 Perla Serfaty-Garzon, "Habiter», in: M. Segaud, J. Brun et J.-C. Driant (dir.), Dictionnaire de l'habitat et du logement, Paris, Armand Colin, 2003, p. 213-214.

25 Bob Rowntree, «Les cartes mentales, outil géographique pour la connaissance urbaine. Le cas d'Angers (Maine-et-Loire)», Norois, n 176 (1997), p. 585-604, ici p. 586. 
2 et 3. Relevé ethno-architectural d'une salle de cours et carte mentale de la ville de Strasbourg
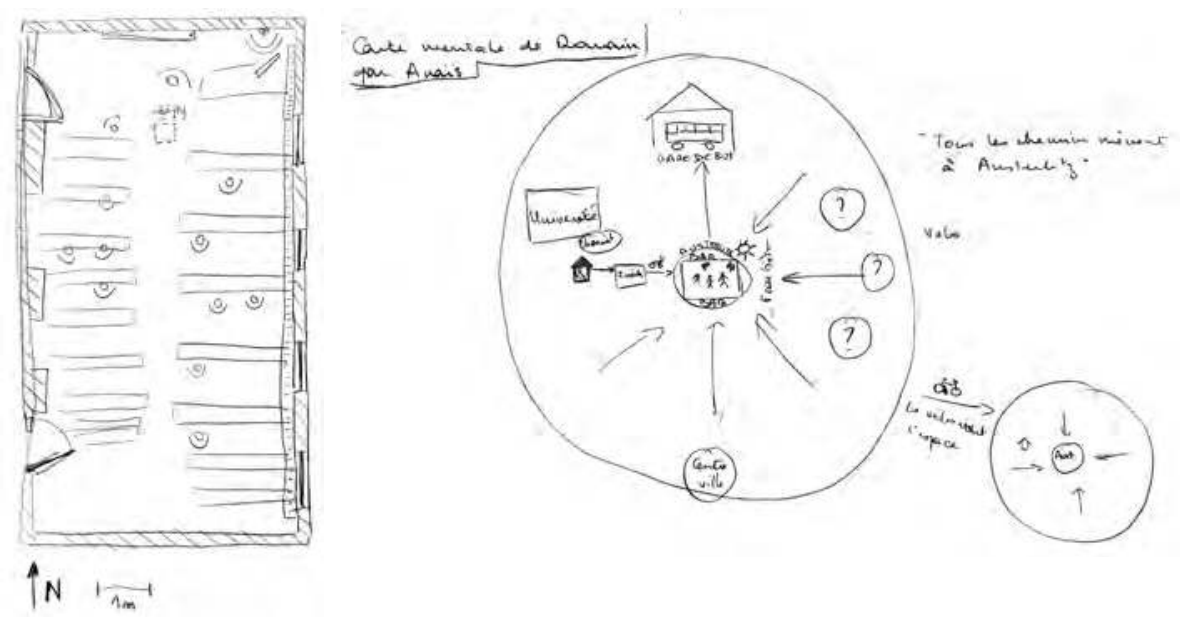

Ainsi, nous avons expérimenté de nombreuses conventions de représentation afin de dépasser la frustration face à l'incapacité à rendre compte du réel: perspective à un ou deux points de fuites, axonométrie, perspective cavalière et isométrique, perspective signifiante (consiste à donner aux personnages une taille proportionnelle à leur importance symbolique, et aux objets et bâtiments une forme qui convient au sens qui leur est associé), mais aussi différentes conventions de représentation de chorégraphies. Au fur et à mesure des séances, des automatismes sont apparus. S'étant ainsi construit une boîte à outils, les participants ont pu se détacher de l'intention de faire beau, et se concentrer sur le contenu, le sens, et la manière la plus efficace de le retranscrire.

\section{Apprendre à sélectionner, ou l'art de l'inachevé}

Un autre réflexe dont il faut s'éloigner dans le cadre du croquis ethnographique est le désir de créer un dessin achevé. Hiérarchiser les informations signifie en représenter certaines et en suggérer d'autres; c'est choisir quelles parties du croquis seront les plus détaillées. Sur le terrain, le temps alloué à chaque croquis est très limité. L'apprentissage de l'organisation du temps est donc un enjeu. Un exercice permettant de s'entraîner à aller à l'essentiel a été proposé aux participants dès les premières séances. C'est celui que nous vous avons proposé en introduction de cet article. L'un des participants prend la pause. Autour, les autres le croquent en un temps limité, qui va en se réduisant de 5 minutes à 10 secondes. Dans cet exercice, on cherche à représenter le corps entier et sa position dans l'espace: en raccourcissant le temps d'exécution d'une pause à l'autre, le trait cherche l'efficacité. Laurane Delavier a demandé à chacun de prêter une attention à la composition du corps du point de vue biologique (le squelette, les muscles, etc.), pour apprendre à comprendre et reproduire les volumes, les proportions et l'articulation des membres, la répartition du poids et l'équilibre du corps. Cet exercice permet de prendre l'habitude de dessiner vite, il aide à comprendre et à placer correctement et rapidement les éléments du dessin. 


\section{4, 5, 6 et 7. Croquis rapides, quatre auteurs différents pour une même pause}

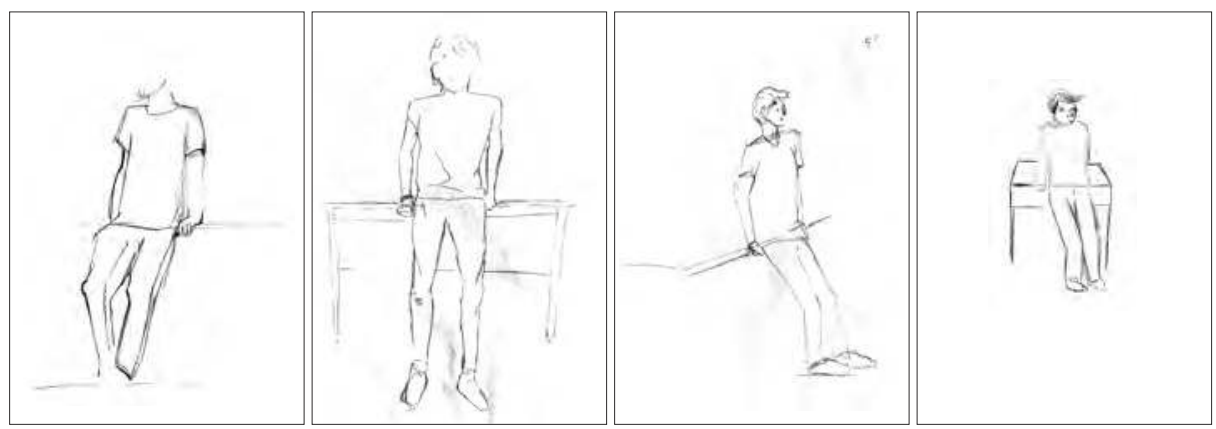

Les croquis ci-dessus ont été dessinés par quatre personnes différentes, lors de la même pause. Pris par le temps et ne maîtrisant pas le dessin, les participants n'ont pas eu conscience d'être en train d'effectuer des choix. C'est une fois finis, en les posant côte à côte afin d'en discuter collectivement, que cela apparaît. Le premier donne des informations sur les vêtements, en peu de traits le tissu est décrit: on comprend qu'il s'agit d'un jean. Mais le visage et les extrémités de l'individu ne sont que suggérés. Le deuxième esquisse les plis du vêtement, n'indiquant rien de leur matière mais informant sur le corps habillé qui prend ainsi du relief. Le troisième paraît très plat mais les traits du visage et les cheveux sont détaillés. Sur le premier, comme sur le troisième, sol et bureau ne sont que des lignes horizontales. Impossible de savoir qu'il s'agit d'un meuble, mais on comprend le rôle de soutien du corps. Sur le deuxième et le quatrième le bureau est identifiable, ses principaux éléments de structure apparaissent clairement. Le spectateur reconstruit alors de lui-même l'emplacement du sol au bas des pieds du bureau. Les éléments représentés, suggérés, ou écartés ne sont pas les mêmes pour ces quatre croquis. Les stratégies de suggestions sont également différentes. Le quatrième croquis permet d'évoquer une limite de la méthode: la surinterprétation. Tout observateur extérieur pourrait être tenté d'y voir la représentation de l'état d'esprit de la personne qui pose: par le langage non verbal - regard fuyant, genoux rentrés -, renforcé par la position du dessin dans la feuille, le sentiment de timidité ou de malaise se dégage du personnage. Mais est-ce réellement ce qu'a voulu dire l'auteur? C'est pour limiter ce risque que nous avons restreint l'utilisation du croquis comme outil d'enquête dans notre atelier: émetteur et destinataire du message sont confondus. Par ailleurs, la relecture par l'auteur du dessin peut faire apparaître à ses yeux des éléments dont il n'avait pas conscience en dessinant, faire émerger de nouveaux intérêts notamment en observant les récurrences dans son propre carnet de croquis ${ }^{(26)}$.

L'inachèvement pose aussi la question du temps. D'une part, le temps de l'action observée. Nous avons constaté que la prise de croquis est bien plus facile lorsque la scène observée est statique, lente ou répétée. Pour Alain Vulbeau, «aussi réductrice et imprécise que soit cette méthodologie en apparence, elle permet de sauver des situations, de leur faire une place et de leur donner un statut: celui d'instantanés

26 Rachel PERREL, «Le croquis ethnographique. L'expérience d'un autre médium par un groupe d'étudiants de l'Université de Strasbourg dans le cadre d'un atelier culturel», Revue des Sciences Sociales, $\mathrm{n}^{\circ} 54$ (2015), p. 40-49, ici p. 44. 
socio-ethnographiques» ${ }^{(27)}$. Ce chercheur fige ainsi un moment. Pour notre part, nous avons au contraire cherché à intégrer la dimension du temps qui passe, du mouvement en train de se faire. C'est là un avantage que le croquis a sur la photographie: une seule image permet d'articuler en elle plusieurs instants, ou décrire une durée. D'autre part, il y a le temps du dessin. Peut-on s'autoriser à compléter un croquis ultérieurement, de mémoire? Nous n'avons pas pris position sur ce point au cours de l'atelier. Cette interrogation rejoint celles autour de la prise de note écrite. Mais, l'écrit étant une modalité de retranscription qui semble aller de soi, il est rare de se poser de telles questions sur les biais de la méthode que le croquis permet de soulever ${ }^{(28)}$.

\section{Conclusion}

Dessin et ethnographie sont toujours allés de pair, mais l'usage du dessin au sein de la discipline a évolué au cours du temps. Le croquis comme méthode d'enquête est relativement récent. Le croquis est une méthode de dessin partagé par plusieurs disciplines. Il devient croquis ethnographique quand il est utilisé dans le cadre de l'enquête de terrain, quand le regard du chercheur et sa façon d'interpréter le monde qui l'entoure par le biais de l'anthropologie font du croquis un outil d'accès à la connaissance et de transmission de celle-ci. C’est cette propriété qui nous a permis de faire des emprunts à d'autres domaines, et d'en faire un outil adapté à notre besoin. Ainsi, la relation au dessin est modifiée par le regard du chercheur, mais inversement, le dessin modifie et oriente son regard porté sur le terrain: la façon d'observer n'est pas la même lorsque les notes dans le carnet de terrain sont sous forme graphiques ou écrites. S'exercer au croquis ethnographique revient donc à développer une habileté visuelle plus que manuelle. Pourtant c'est cette dernière qui a été à l'origine des appréhensions formulées par les participants de l'atelier, issues du désir de bien dessiner. Pour dépasser cette hésitation, il faut comprendre que le croquis est un moyen de communication avant tout. Qu'il est constitué de codes, que nous avons appris à manipuler. Qu'il sert à transcrire une expérience vécue, et qu'il porte en lui les hypothèses d'analyse qu'en fait le chercheur.

Être déstabilisé par la méthode permet de la questionner continuellement. Dans cet article, comme dans les ateliers, nous nous sommes appuyés sur des comparaisons avec d'autres méthodes pour comprendre les avantages et inconvénients du croquis. Voici certains avantages relevés collectivement: il demande du matériel et des connaissances techniques limités; il est rapide à exécuter et à relire; il permet de donner des informations que les autres méthodes ne permettent pas, ou mal, de retranscrire; il inclut déjà des éléments d'analyse, des hypothèses; il permet une relation plus fluide sur le terrain avec les interlocuteurs; il peut être un support d'échange, surtout dans un contexte d'échanges en langue étrangère. Nous avons également listé ses limites: la difficulté à retranscrire la réalité, l'imprécision du dessin; le fait qu'il n'enregistre pas ce que le dessinateur n'a pas vu, comme pourrait le faire une photographie; le risque de surinterprétation; le risque de dérive vers la fiction. Néanmoins, certaines de ces

27 Alain Vulbeau, «Côte à côte en ville: analyse de 20 situations de prises de mains ", Spécificités, $\mathrm{n}^{\circ} 7$ (2015), p. 7-26, ici p. 12.

28 R. PERrel, « Le croquis ethnographique» (note 26), ici p. 47. 
limites ne sont pas propres à cette méthode et font appel à l'honnêteté intellectuelle de chacun. Comparer les méthodes ne veut pas dire qu'elles soient mutuellement exclusives, bien au contraire. Le croquis s'avère particulièrement complémentaire à la prise de notes écrites, dont il partage les outils. La page de carnet ci-dessous montre bien cette imbrication entre esquisse en perspective, plan schématique, phrases et motsclés pour apporter différents éclairages sur une scène.

À la fin de l'année universitaire, nous avons pu observer que notre propre utilisation du croquis avait évolué, et que les pages des carnets des étudiants ayant participé aux ateliers s'étaient enrichies de dessins, schémas et esquisses. Ils ont modifié leur

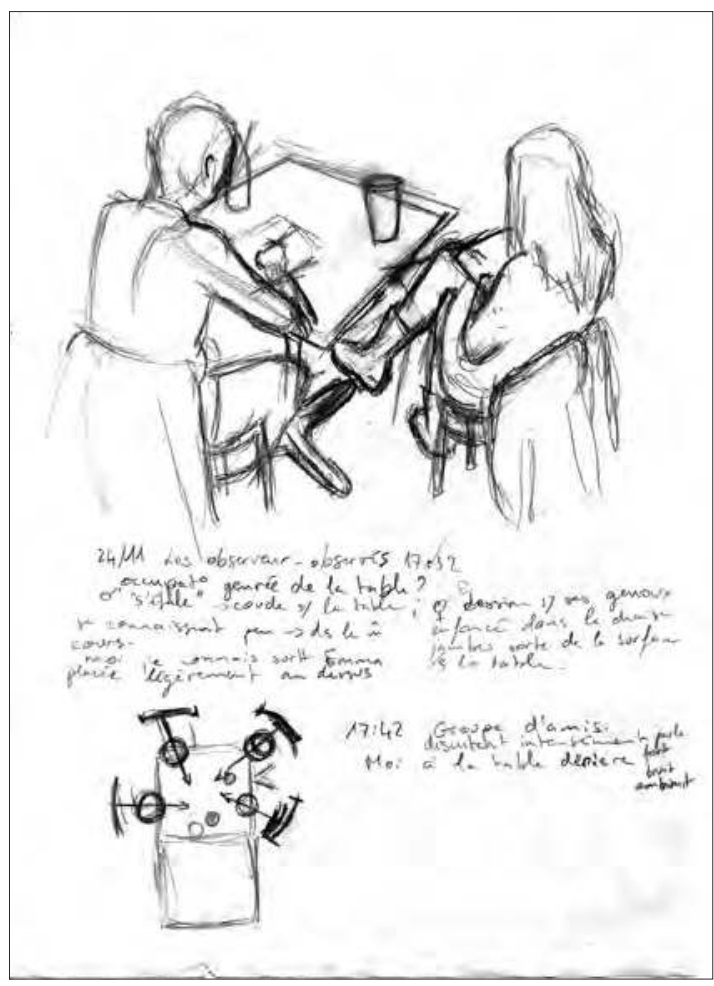
relation au dessin, mais surtout ils ont conservé ce regard spécifique pour croquer. L'atelier a engendré un nouvel intérêt pour le dessin ethnographique au sein de l'université de Strasbourg, qui, nous l'espérons, sera transmis aux prochaines générations d'étudiants. Cela semble être sur la bonne voie, l'atelier ayant été renouvelé l'année scolaire suivante, bâti dans la continuité de la première édition mais porté par de nouveaux acteurs.

8. Page d'un carnet de terrain, observation de l'occupation de l'espace dans un bar

\section{Résumé}

Un atelier de croquis ethnographique sur le thème "Le corps dans l'espace» a été mis en place, sur une initiative étudiante, à l'Institut d'ethnologie de l'université de Strasbourg au courant de l'année scolaire 2016-2017. Cet article est un retour d'expérience de cette activité. Il reprend et prolonge certaines réflexions menées par le groupe autour de la question du dessin comme mode de production du savoir valide. Afin de construire un outil adapté à nos besoins, l'atelier a été nourri de méthodes issues des arts plastiques, de l'architecture et de l'archéologie. Le croquis ethnographique est d'une part un regard spécifique sur le monde, une habilité visuelle exercée par le chercheur. D’autre part, il est un outil de communication, servant à transcrire une expérience vécue et portant en lui les hypothèses d'analyse qu'en fait le chercheur. 


\begin{abstract}
An ethnographic sketch workshop on "The Body in Space" was set up, on a student initiative, at the Institute of Ethnology of the University of Strasbourg during 2016-2017. This article is a feedback from this activity. It takes up and extends some reflections led by the group around the question of drawing as a valid mode of production of knowledge. In order to build a tool adapted to our needs, the workshop was nourished with methods from the visual arts, architecture and archeology. The ethnographic sketch is on the one hand a specific look at the world, a visual skill exerted by the researcher. On the other hand, it is a communication tool, used to transcribe an experience and carrying in it the analysis hypotheses made by the researcher.
\end{abstract}

\title{
Zusammenfassung
}

Auf studentische Initiative wurde 2016/2017 am ethnologischen Institut der Universität Strassburg ein ethnologischer Skizzen-Workshop zum Thema „Körper im Raum" angeboten. Dieser Artikel berichtet über diese Erfahrung. Die Überlegungen der Gruppe um die Frage des Zeichnens als Art einer gültigen Wissensproduktion werden aufgenommen und vertieft. Um eine unserer Bedürfnisse entsprechende Herangehensweise zu schaffen wurde der Workshop durch Methoden der Bildenden Künste, der Architektur und der Archeologie bereichert. Die ethnologische Skizze ist zum einen ein spezifischer Blick auf die Welt, eine vom Forscher geübte visuelle Kunstfertigkeit. Zum zweiten ist sie ein Kommunikationswerkzeug, die der Übertragung des Erlebten dient und die die Hypothesen des Forschers beinhaltet. 